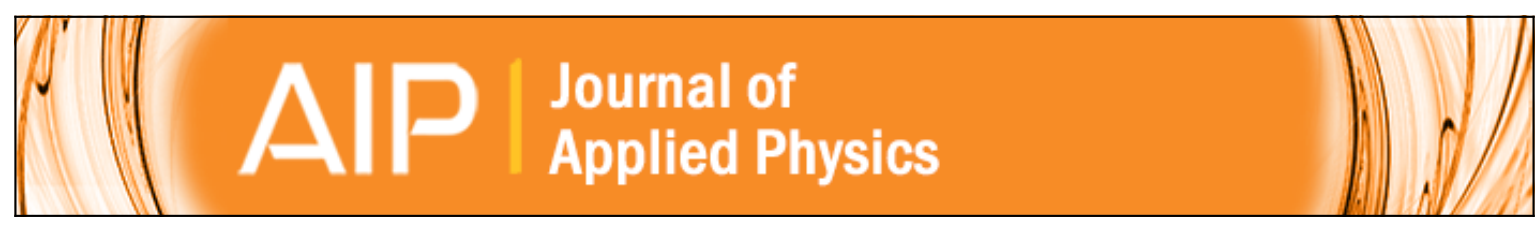

\title{
Anisotropic magnetism in hybridizing uranium systems
}

N. Kioussis, H. J. Yu, B. R. Cooper, Q. G. Sheng, and J. M. Wills

Citation: Journal of Applied Physics 73, 5424 (1993); doi: 10.1063/1.353703

View online: http://dx.doi.org/10.1063/1.353703

View Table of Contents: http://scitation.aip.org/content/aip/journal/jap/73/10?ver=pdfcov

Published by the AIP Publishing

\section{Articles you may be interested in}

$\mathrm{Ab}$ initio study of anisotropic magnetism in uranium compounds

J. Appl. Phys. 85, 6226 (1999); 10.1063/1.370229

First principle evaluation of hybridization and exchange effects for magnetic ordering in correlated-electron uranium systems

J. Appl. Phys. 70, 6083 (1991); 10.1063/1.350053

Synthesis of band and model Hamiltonian theory for strongly hybridizing uranium systems

J. Appl. Phys. 67, 5197 (1990); 10.1063/1.344660

Theory of strong hybridization-induced relaxation in uranium systems

J. Appl. Phys. 64, 5592 (1988); 10.1063/1.342291

Two-ion anisotropic interaction from band f electron hybridization and magnetic ordering in uranium intermetallics

J. Appl. Phys. 53, 7902 (1982); 10.1063/1.330381

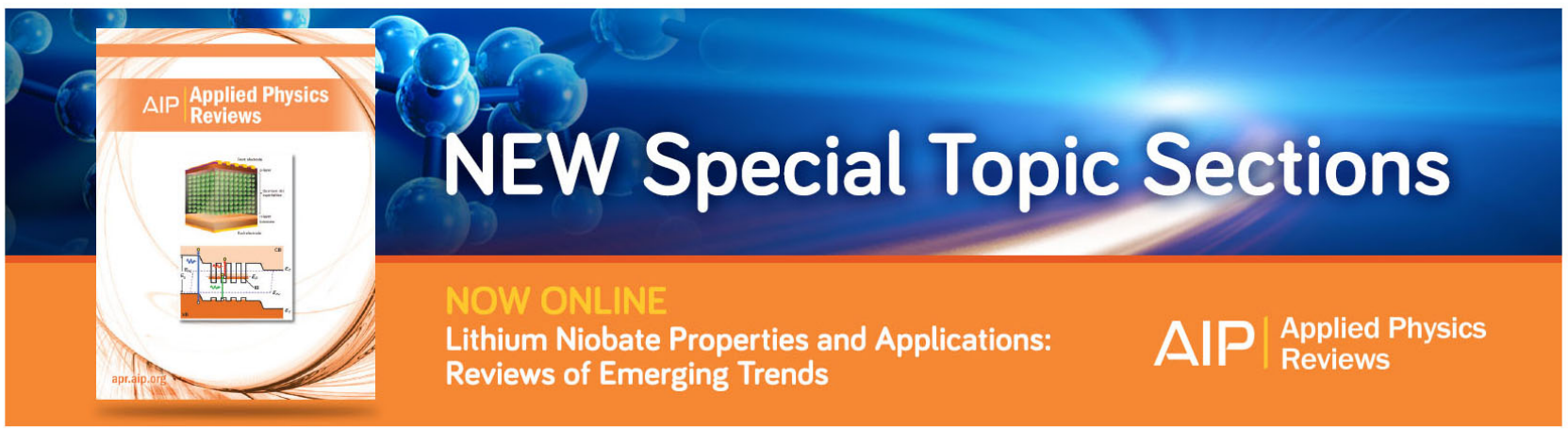




\title{
Anisotropic magnetism in hybridizing uranium systems
}

\author{
N. Kioussis and H. J. Yu \\ Department of Physics, California State University at Northridge, Northridge, California 91330
}

B. R. Cooper and Q. G. Sheng

Department of Physics, West Virginia University, Morgantown, West Virginia 26506

J. M. Wills

Los Alamos National Laboratory, Los Alamos, New Mexico 87545

The isostructural uranium monopnictides and monochalcogenides have become prototype systems in actinide research with respect to their unusual magnetic properties. We have investigated the origins in the electronic structure of the variation in magnetic behavior as the degree of $5 f$-electron localization changes from localized to itinerant on going up the pnictogen or chalcogen column, thus decreasing the U-U separation. We have applied a synthesis of: (1) A phenomenological theory of orbitally driven magnetic ordering which includes both the hybridization-induced and the RKKY exchange interactions on an equal footing, and (2) $A b$ initio electronic structure calculations, based on the linear-muffin-tin-orbital method, allowing a first-principles evaluation of the parameters entering the model Hamiltonian. We have investigated systematically characteristic trends and changes of the $5 f$-state resonance width, the hybridization potential, and the hybridization-induced and RKKY exchange interactions with chemical environment, on going down the pnictogen or chalcogen column and on going from the weakly hybridizing pnictides to the more strongly hybridizing chalcogenides.

\section{INTRODUCTION}

Among the actinide compounds, the uranium monopnictides $\mathrm{UX}(\mathrm{X}=\mathrm{P}, \mathrm{As}, \mathrm{Sb}, \mathrm{Bi})$ and monochalcogenides $\left(\mathrm{X}=\mathrm{S}, \mathrm{Se}, \mathrm{Te}\right.$ ) have become ${ }^{1}$ prototype systems in actinide research with respect to their unusual magnetic properties and electronic structure. This class of isostructural compounds, with the simple rock-salt structure, offers ${ }^{2}$ the opportunity to continuously vary the U-U spacing from $4.5 \AA$ in UBi to $3.4 \AA$ in UN in the pnictide series or from $4.2 \AA$ in UTe to $3.9 \AA$ in US in the chalcogenide series, and to investigate, in turn, the change of magnetic behavior as the degree of the $5 f$-electron localization changes from localized (correlated ionic) to itinerant behavior. Further, this family of systems allows one to examine systematically the effects of hybridization between the $\mathrm{U}^{3+} 5 f$ and uranium $6 d$ or ligand $p$ electrons on the change of magnetic behavior on going from the monopnictides to the monochalcogenides of uranium, both of which have rock-salt structure and where the only obvious change is the addition of an anion $p$ electron.

The onset of magnetic ordering in this class of compounds typically has behavior characteristic of twodimensional systems although these systems are cubic of rock-salt structure. ${ }^{3}$ Thus, one of the central features of the anomalous behavior is the presence of an extremely large anisotropy. The uranium monopnictides order with the type-I antiferromagnetic structure, in which ferromagnetic (001) planes are stacked antiferromagnetically into a $(\uparrow \downarrow \uparrow \downarrow)$ sequence along the $\langle 001\rangle$ direction and moments perpendicularly to the planes. ${ }^{3}$ The Néel temperature and low temperature moment increase steadily from UN ( $53 \mathrm{~K}$ and $0.75 \mu_{B}$ ) to UBi $\left(285 \mathrm{~K}\right.$ and $\left.3.0 \mu_{B}\right){ }^{4}$ On the other hand, the uranium monochalcogenides provide an interesting sequence of metallic ferromagnets which exhibit strong magnetic anisotropy along the $\langle 111\rangle$ direction rather than the cube edge as observed in the uranium pnictide series. ${ }^{5,6}$ While the low temperature moment increases from US $\left(1.7 \mu_{B}\right)$ to UTe $\left(2.2 \mu_{B}\right)$, the Curie temperature decreases with increasing lattice constant (from $180 \mathrm{~K}$ in US to 104 $\mathrm{K}$ in $\mathrm{UTe}$ ), a rather surprising result in view of the increasing uranium-uranium separation on going down the chalcogen column., ${ }^{5,6}$ Despite the well-defined magnetic ordering ${ }^{3,4,6}$ in these compounds, no sharp collective excitations or crystal-field transitions below $40 \mathrm{meV}$ have been observed in inelastic neutron scattering experiments ${ }^{5,6-8}$ in these systems except for USb and UTe, but rather there is a continuous broad spectrum of magnetic response. Even when sharp collective excitations are observed in USb and $\mathrm{UTe},{ }^{5-8}$ these are imposed on a broad spectrum and they tend to disappear well below the ordering temperature.

In spite of the considerable research effort undertaken $^{1-8}$ on this class of systems, no clear theoretical understanding of their electronic structure and unusual magnetic behavior has emerged. ${ }^{5}$ The difficulty stems from the more extended, spatially diffuse, nature of the $5 f$ electrons and their ability to hybridize strongly with the actinide $6 d$ and anion $p$ electrons, giving rise to a large variety of complex magnetic and electronic behavior. The purpose of this paper is to investigate the origins, in the electronic structure, of the variation of the hybridization-induced magnetism with chemical environment on going down the pnictogen or chalcogen column, increasing thus the degree of $5 f$-electron localization as the U-U separation increases, and on going from the weakly hybridizing pnictides to the more strongly hybridizing chalcogenides. We have employed a unified theory ${ }^{9-13}$ that we have developed over a period of time, which involves (1) a phenomenological theory of moderately delocalized $f$-electron systems which treats both the band-f hybridization and the band-f cou- 


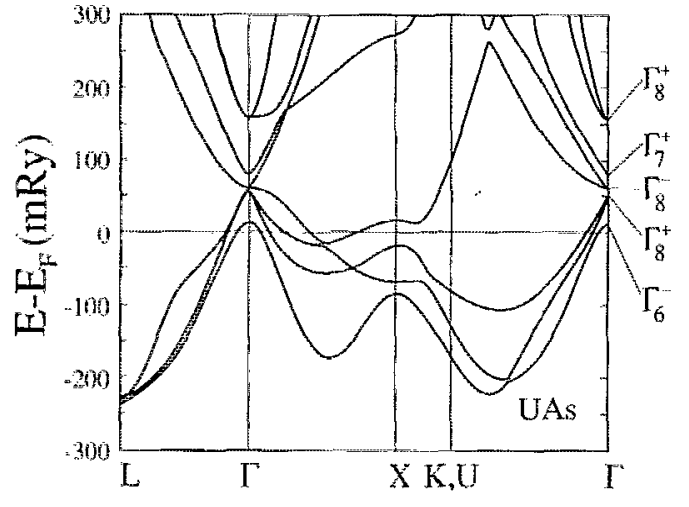

FIG. 1. The band strncture of UAs, calculated with the U $5 f$ states treated as core states, along symmetry lines in the Brillouin zone. The band energies are refative to the Fermi energy. The labels on the right of the figure denote the symmetry of the bands at $\Gamma$. Anion $p$-derived bands have $\Gamma_{t}$ or $\Gamma_{\overline{8}}^{-}$symmetry, while $\mathrm{U} 6 d$-derived bands have $\Gamma_{7}^{+}$and $\Gamma_{\tilde{x}}^{+}$ symmetry.

lomb exchange on an equal footing, ${ }^{12,13}$ and (2) ab initio electronic structure calculations, based on the linearmuffin-tin-orbital (LMTO) method, ${ }^{14}$ allowing a firstprinciples evaluation of the parameters entering the phenomenological theory.

As the formal aspects of the theory have been reviewed in previous work, ${ }^{9-13}$ we shall forgo all but a brief description of this method here. The self-consistent one-electron potential is obtained ${ }^{10,11}$ from a warped-muffin-tin LMTO calculation without recourse to the atomic sphere approximation, ${ }^{14}$ i.e., using a nonzero tail parameter as a variational parameter. The full interstitial is used and the only shape approximation to the potential is a spherical averaging in nonoverlapping muffin-tin spheres. The uranium $5 f$ electrons are treated as localized rather than itinerant states and thus are included self-consistently as core states at each iteration, not being allowed to hybridize with band states. The relative magnitudes of the muffin-tin spheres radii are chosen so that nearest-neighbor muffin-tin spheres touch at the minimum in the charge density between nearest neighbors. The electronic structure calculation provides the band states and energies and a self-consistent hybridization potential, thereby allowing determination of the hybridization matrix elements, $V_{\mathrm{k} m}$, and the band- $f$ coulomb exchange matrix elements, $J_{n n^{\prime}}\left(\mathbf{k}, \mathbf{k}^{\prime}\right)$. The $5 f$-state energy relative to the Fermi energy $E_{f}-E_{F}$, and the intra-atomic Coulomb energy $\mathrm{U}$, are determined from supercell calculations, ${ }^{11}$ treating one uranium ion out of four as a defect differing from the nominal configuration by the addition or subtraction of an electron. Having determined the phenomenological model Hamiltonian parameters on an absolute basis, one can evaluate ${ }^{9-13}$ the hybridization-mediated and RKKY two-ion exchange interactions, responsible for the unusual magnetic ordering in this class of systems.

\section{RESULTS AND DISCUSSION}

The band structure of UAs (typical of the U monopnictides), calculated with the $U 5 f$ states treated as core states, is plotted along symmetry lincs in Fig. 1, with the

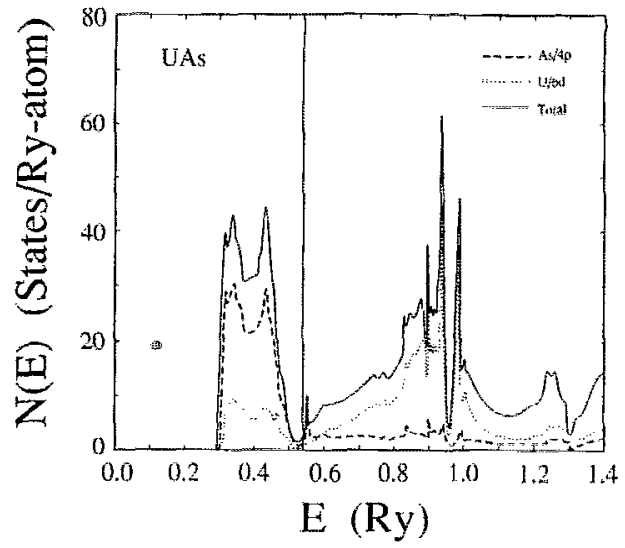

FIG. 2. Total density of states (solid curve) of UAs corresponding to the band structure in Fig. 1. Also shown are the partial density of states for the $\mathrm{U} d$-derived (dotted curve) and As $p$-derived bands (dashed curve), respectively.

band energies measured with respect to the Fermi energy. At $\Gamma$, the $U 6 d$-derived bands are the bases for three representations, one with $\Gamma_{7}{ }^{(+)}$and two with $\Gamma_{8}{ }^{(+)}$symmetry, while anion $p$-derived bands at $\Gamma$ have $\Gamma_{6}{ }^{(-)}$and $\Gamma_{8}^{(-)}$symmetry. The bands dominating the hybridizationinduced properties are largely derived from anion $p$ states and uranium $6 d$ states. The $p$-derived bands at $\Gamma$ of $\Gamma_{8}^{(-)}$ symmetry move closer to the Fermi energy on going up the pnictogen column, indicating an increase of the $p-f$ hybridization as the uranium-anion separation decreases. The addition of an anion $p$ electron on going from the monopnictides to the monochalcogenides of uranium causes the $p$-derived bands to sink far below the Fermi energy, so that the bands dominating the hybridization-induced properties in the chalcogenide series are largely derived from the $\mathrm{U} 6 d$ states. The total density of states corresponding to the band structure in Fig. 1 is shown in Fig. 2 (solid curve). Also plotted in the same figure is the partial density of states of the $U d$-derived bands (dotted curve) and the As $p$-derived bands (dashed curve), respectively. In all the $U$ monopnictides the Fermi energy lies in a low-density-of-states energy region between bands of predominantly pnictogen $p$ character and $\mathrm{U} d$ character, respectively. On the other hand, the Fermi energy in the $U$ monochalcogenides lies in a high-density-of-states energy region, which are largely derived from $\mathrm{U} 6 d$ states.

Calculated results of the $U$ muffin-tin radius, the Fermi energy, $E_{F}$, the $5 f$ resonant energy $E_{5 f}$, the total and partial $U d$ - and anion $p$-derived density of states at $E_{F}$ the value of the $5 f$ wave function $\varphi_{f}(s)$ at the $U$ muffin-tin radius, the $f$-state resonance width $\Gamma$ characterizing the strength of the hybridization, ${ }^{10,11}$ and the hybridization potential $v\left(\kappa_{F}\right),{ }^{10,11}$ are listed in Table $\mathrm{I}$, for the uranium monopnictides and monochalcogenides, respectively. As the degree of $f$-electron localization decreases $\left[\varphi_{f}(s)\right.$ increases] on going up the pnictogen or chalcogen column, the resonance width increases, and consequently the strength of the $f$-band hybridization. This results from an increase of $f$ hybridization with $6 d$ clectrons on other ura- 
TABLE I. Summary of the LMTO results for the Fermi energy $E_{F}$, the U $5 f^{3}$ resonance energy $E_{5 g}$ the total density of states $N\left(E_{F}\right)$ at $E_{F}$, the $\mathrm{U}$ $d$-derived $N_{d}\left(E_{F}\right)$ and anion $p$-derived $N_{\rho}^{X}\left(E_{F}\right)$ partial density of states at $E_{F}$, the $5 f$ wave function $\varphi_{i}(s)$, the $5 f$ resonance width $\Gamma$, and the hybridization potential $v\left(\kappa_{F}\right)$, for the $\mathrm{UX}(\mathrm{X}=\mathrm{Bi}, \mathrm{Sb}, \mathrm{As}, \mathrm{P}, \mathrm{Te}, \mathrm{Se}, \mathrm{S})$ isostructural series. Also tabulated are values of the lattice constant, $a$, and the $\mathrm{U}$ muffin-tin radius, $s_{\mathrm{H}}$, in atomic units, respectively; $E_{F}$ and $E_{s f}$ are in $\mathrm{Ry}$, the DOS are in states/Ry, and $\Gamma$ and $u\left(\kappa_{F}\right)$ are in mRy.

\begin{tabular}{|c|c|c|c|c|c|c|c|}
\hline$a$ & 12.056 & 11.711 & 10.899 & 10.562 & 11.631 & 10.847 & 10.374 \\
\hline$s_{t}$ & 2.916 & 2.855 & 2.735 & 2.682 & 2.832 & 2.719 & 2.642 \\
\hline$E_{5 f}$ & 0.484 & 0.535 & 0.648 & 0.712 & 0.560 & 0.667 & 0.754 \\
\hline$N\left(E_{F}\right)$ & 3.49 & 3.05 & 2.17 & 1.88 & 13.64 & 11.38 & 10.38 \\
\hline$N_{d}^{U}\left(E_{F}\right)$ & 1.36 & 0.89 & 0.49 & 0.31 & 8.36 & 6.76 & 5.66 \\
\hline$\Gamma$ & 10.43 & 11.25 & 16.29 & 19.11 & 18.78 & 27.56 & 34.32 \\
\hline$v\left(\kappa_{F}\right)$ & -4.20 & -4.53 & -5.86 & -6.56 & -5.62 & -7.28 & -8.50 \\
\hline
\end{tabular}

nium sites and $p$ electrons on nearest-neighbor pnictogen or chalcogen sites, as the uranium-uranium separation decreases with decreasing anion size. Further, the band- $f$ hybridization, as measured by $\Gamma$, increases by about a factor of 2 on moving from the weakly hybridizing monopnictides to the more strongly hybridizing monochalcogenides of uranium. In the monopnictide series the density of states at $E_{F}, N\left(E_{F}\right)$, which are derived from $\mathrm{U} 6 d$ and pnictogen $p$ states, are by a factor of $3-4$ smaller than the corresponding $N\left(E_{F}\right)$ in the chalcogenide series, which are largely derived from the $\mathrm{U} 6 d$ states. The energy necessary to place a uranium $5 f$ electron in a band state at the Fermi energy, $E_{F}-E_{f}$, and the energy required to change from a $5 f^{3}$ configuration to a $5 f^{4}$ configuration, $E_{f}+U-E_{F}$, have been evaluated from self-consistent supercell calculations. We find that $E_{F}-E_{f}$ and $\mathrm{U}$ are 1.0 and $3.9 \mathrm{eV}$ in $\mathrm{UBi}$, respectively. For both the uranium monopnictide and monochalcogenide series, hybridization rather than coulomb exchange is the larger contributor to the highly anisotropic effective two-ion exchange interaction which determines the magnetic ordering behavior; while for the $\mathrm{CeSb}$ and $\mathrm{CeTe}$ the opposite was found ${ }^{12}$ to be true, with coulomb exchange being dominant in determining the twoion interaction. The stronger hybridization in uranium compounds results from the higher degree of delocalization of the uranium $5 f$ states compared to that of the cerium $4 f$ states. Work currently in progress is aimed at the evaluation of the hybridization-mediated two-ion exchange parameters; and their effect on the magnetic ordering in this class of systems, by taking into account explicitly in the phenomenological Hamiltonian the overlap of the tails of the $5 f$ states on neighboring uranium muffin-tin spheres.

\section{ACKNOWLEDGMENTS}

The research at California State University Northridge (CSUN) was supported by the National Science Foundation under Grant No. DMR-89-18887 and the CSUN Office of Research and Sponsored Projects, and that at West Virginia University by the NSF under Grant No. DMR88-07523. The research at Los Alamos National Laboratory was supported by the U.S. Department of Energy.

'H. Rudiger, H. R. Ott, and O. Vogt, Physica B 130, 538 (1985).

${ }^{2}$ B. Reihl, N. Martensson, and O. Vogt, J. Appl. Phys. 53, 2008 (1982).

${ }^{3}$ G. H. Lander, S. K. Sinha, D. M. Sparlin, and O. Vogt, Phys. Rev. Lett. 40, 523 (1978); B. Hälg and A. Furrer, J. Appl. Phys. 55, 1860 (1984). ${ }^{4}$ S. K. Sinha, G. H. Lander, S. M. Shapiro, and O. Vogt, Phys. Rev. 23, 4556 (1981).

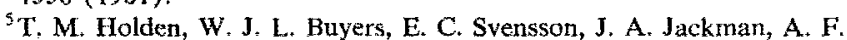
Murray, O. Vogt, and P. de. V. DuPlessis, J. Appl. Phys. 53, 1967 (1982).

${ }^{6}$ T. M. Holden, W. J. L. Buyers, P. de. V. DuPlessis, K. M. Hughes, and M. F. Collins, J. Magn. Magn. Mater. 54-57, 1175 (1986).

'G. H. Lander and W. G. Stirling, Phys. Rev. B 21, 436 (1980); T. M. Holden, W. J. L. Buyers, E. C. Svensson, and G. H. Lander, ibid. 30, 114 (1984).

${ }^{8}$ W. J. L. Buyers, A. F. Murray, T. M. Holden, E. C. Svensson, P. de. V. DuPlessis, G. H. Lander, and O. Vogt, Physica B 102, 291 (1980).

${ }^{9}$ B. R. Cooper, R. Siemann, D. Yang, P. Thayamballi, and A. Banerjea, in Handbook on the Physics and Chemistry of the Actinides, edited by A. J. Freeman and G. H. Lander (North-Holland, Amsterdam, 1985), Vol 2, Chap. 6, pp. 435-500.

${ }^{10}$ J. M. Wills and B. R. Cooper, Phys. Rev. B 36, 3809 (1987).

"N. Kioussis, B. R. Cooper, and J. M. Wills, Phys. Rev. B 44, 10003 (1991).

"'Q. G. Sheng and B. R. Cooper, J. Appl. Phys. 69, 5472 (1991); Q. G. Sheng and B. R. Cooper, ibid. 70, 6083 (1991).

${ }^{13}$ B. R. Cooper, Q. G. Sheng, S. P. Lim, C. Sanchez-Castro, N. Kioussis, and J. M. Wills, J. Magn. Magn. Mater. 108, 10 (1992).

${ }^{14}$ O. K. Andersen, Phys. Rev, B 12, 3060 (1975). 